\title{
REFORMA EDUCATIVA Y POLÍTICAS DE EVALUACIÓN EN MÉXICO, ¿INSTRUMENTOS PARA ABATIR EL REZAGO ESCOLAR Y PROMOVER LA IGUALDAD DE OPORTUNIDADES?
}

\author{
EDUCATION REFORM AND EVALUATION POLICIES \\ IN MEXICO: INSTRUMENTS FOR OVERCOMING \\ THE LEARNING GAP AND PROMOTING EQUAL \\ OPPORTUNITIES
}

\section{LA RÉFORME DE L'ENSEIGNEMENT ET LES POLITIQUES D'ÉVALUATION AU MEXIQUE SONT-ELLES UTILES \\ À SURMONTER LE RETARD SCOLAIRE ET À PROMOUVOIR L'ÉGALITÉ DES CHANCES?}

\author{
LAURA FLAMAND \\ El Colegio de México \\ lflamand@colmex.mx \\ RAFAel Arriaga \\ El Colegio de México \\ racarrasco@colmex.mx \\ Claudia Santizo \\ Universidad Autónoma Metropolitana \\ crodall@yahoo.com
}

Resumen: Con el propósito de desarrollar políticas públicas basadas en evidencia, este artículo se ocupa de comparar las maneras en que se evaluaba la política educativa antes y después de la reforma educativa de 2013. Nos concentramos en tres temas cruciales en la evaluación: logro en aprendizajes, evaluación a docentes y programas federales de educación. El análisis comparado rastrea los cambios clave que experimentó la evaluación en cuatro rubros: la toma de decisiones, los actores, los objetos específicos de la evaluación y los métodos. Concluimos que la información que generó el Sistema Nacional de Evaluación Educativa (SNEE) no contribuyó a decisiones que promovieran la 
calidad de la educación y revelamos la influencia persistente de la Secretaría de Educación Pública (SEP), así como la creciente participación de los gobiernos estatales en el SNEE.

Palabras clave: reforma educativa; evaluación de política educativa; Instituto Nacional para la Evaluación de la Educación; calidad; igualdad.

Aвstract: With the aim of developing evidence-based public policies, this paper seeks to compare the ways education policy has been evaluated before and after the 2013 education reform. It focuses on three crucial aspects of the evaluation: learning achievements, teacher evaluation and federal education programs. The comparative analysis tracing the key changes undergone by the evaluation process in four areas: decision making, stakeholders, specific objects of evaluation, and methods. We conclude that the information generated by the National System for Education Evaluation (SNEE) did not contribute to decisions that improved the quality of education, and we reveal the continued influence of the Ministry of Education (SEP) as well as the growing involvement of state governments in the SNEE.

Keywords: education reform; evaluation of education policy;

National Institute for Education Evaluation; quality; equality.

\section{Traducción de Gonzalo Celorio Morayta}

Résumé: Appuyé sur l'évidence disponible et dans l'espoir de contribuer au développement dans ce domaine, l'article compare les façons d'évaluer la politique éducative avant et après la réforme de l'enseignement de 2013. L'attention est centrée sur trois sujets essentiels: les progrès en matière d'apprentissage, l'évaluation des enseignants et les programmes fédéraux pour l'éducation. Une analyse comparative met en relief les changements des modes d'évaluation, qui ont lieu sur quatre plans: la prise de décision, les acteurs, les objets précis à évaluer et les méthodes. Notre conclusion est que l'information recueillie par le Système national d'évaluation éducative (SNEE) n'a point conduit à des mesures pour améliorer la qualité de l'enseignement. De même, nous examinons l'influence constante du ministère de l'Éducation (SEP) et la participation croissante des gouvernements des entités fédératives au SNEE. 
Mots clefs: Réforme de l'enseignement; évaluation de la politique éducative; Institut National pour l'Évaluation de l'Enseignement; qualité; égalité.

\section{Traducción de BERNARdo Mabire}

Fecha de recepción: junio de 2019

Fecha de aceptación: noviembre de 2019

\section{INTRODUCGIÓN}

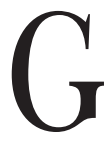

arantizar, o incluso proveer de manera directa, educación de calidad a todas las personas que habitan un país es una de las principales responsabilidades de los gobiernos en el siglo xxi. Una comunidad educada es el motor indispensable para el desarrollo económico, político y social de una nación. ${ }^{1}$ La educación, además, se ha revelado como un poderoso instrumento de movilidad social, aunque diversos trabajos han demostrado que también puede reproducir la desigualdad. ${ }^{2}$ Con estas consideraciones, en la última década, numerosos gobiernos nacionales y diversas organizaciones internacionales han propuesto que las metas centrales de la política educativa sean incrementar su calidad y abatir las desigualdades vinculadas al derecho universal a la educación. ${ }^{3}$ ¿Cómo garantizar que las políticas educativas avancen de manera efectiva hacia estas metas?

${ }^{1}$ Fabian Lange y Robert Topel, "The Social Value of Education and Human Capital", en Eric Hanushek y Finis Welch (eds.), Handbook of the Economics of Education, vol. 1, Países Bajos, Elsevier, 2006, pp. 459-509.

2 Pierre Bourdieu y Jen-Claude Passeron, Reproduction in Education, Society and Culture, $2^{\mathrm{a}}$ ed., Londres, Sage Books, 1990.

${ }^{3}$ Por ejemplo, Organización de las Naciones Unidas (ONU), Objetivos de Desarrollo Sostenible, 2017, http://www.undp.org/content/undp/es/ home/sustainable-development-goals.html (consulta del 8 de enero de 2018), y Organización de las Naciones Unidas para la Educación, la Cien- 
En los planes nacionales de desarrollo de los presidentes Felipe Calderón y Enrique Peña Nieto es posible observar continuidad en dos objetivos de la política educativa: mejorar la calidad e impulsar la equidad. Diversos estudios señalan que, aunque ha habido avances en la equidad y la gerencia del sistema educativo, la calidad sigue siendo deficiente. ${ }^{4}$ En la evaluación más reciente del Programa Internacional para la Evaluación de los Estudiantes (PISA por sus siglas en inglés) en México, los alumnos que lograron buenos aprendizajes -aquellos en el nivel tres o superiores a la escala de seis- son apenas de $17 \%$ en ciencias y matemáticas, y de $25 \%$ en lenguaje. ${ }^{5}$ Respecto a la equidad, aumentar la cobertura en educación fue la estrategia común en ambos planes, con medidas encaminadas a la matrícula escolar. Ambas administraciones se propusieron, además, no dejar fuera a grupo poblacional alguno, poniendo en marcha programas específicos de inclusión educativa. Sin embargo, en 2014 una persona joven del quintil más alto de ingresos tenía una probabilidad $23 \%$ mayor de acceder a la secundaria que una del quintil más bajo. ${ }^{6}$ ¿Cómo mejorar las intervenciones gubernamentales en México para incrementar la calidad educativa y abatir las desigualdades?

cia y la Cultura (UNESCO), Changing dynamics in the governance of education: A comparative analysis of emerging issues and reforms in emerging countries, 2016, http:/ / www.unesco.org/new/fileadmin/MULTIMEDIA/HQ/ED/pdf/ report-watanabe.pdf (consulta del 8 de enero de 2018). Los 17 Objetivos de Desarrollo Sostenible (oDs) son un llamado para adoptar medidas que acaben con la pobreza, protejan al planeta y garanticen que todas las personas gocen de paz y prosperidad. El cuarto objetivo es proveer educación de calidad, mientras que el décimo se refiere a reducir las desigualdades.

${ }^{4}$ Carlos Ornelas, "The Politics of Privatization, Decentralization and Education Reform", en Joseph Zajda (ed.), Decentralization and Privatisation in Education. The Role of the State. Países Bajos, Springer, 2006, pp. 207-228.

${ }^{5}$ El Colegio de México, Desigualdades en México / 2018, México, El Colegio de México, 2018, p. 36.

6 Idem. 
Alrededor del mundo y de manera reciente, la práctica y los recursos destinados a evaluar las intervenciones públicas ha aumentado de manera muy considerable con el propósito de desarrollar acciones a partir de evidencia concreta en cuanto a su capacidad para, por ejemplo, incrementar la calidad educativa o promover la igualdad. ${ }^{7}$ La evaluación de los procesos, los resultados y los efectos de las políticas, programas o acciones gubernamentales puede proporcionar información vital para diseñar e implementar intervenciones públicas más eficientes.

La evaluación sólo puede orientar la acción pública de manera exitosa si es rigurosa en términos conceptuales y metodológicos. El rigor de la evaluación no depende únicamente de la capacidad de los equipos encargados de realizarla, también son necesarias condiciones institucionales que garanticen la continuidad de los esfuerzos y la autonomía de los procesos de evaluación. De acuerdo con Felipe Martínez y Emilio Blanco, ${ }^{8}$ es indispensable un marco normativo adecuado, así como el autocontrol de las autoridades educativas que pueden estar tentadas a politizar el proceso de evaluación, en particular en el caso mexicano. Asimismo, para garantizar la utilidad de las evaluaciones es importante que el diseño, la implementación y el análisis estén abiertos tanto al escrutinio público como a la revisión de especialistas independientes.

Durante el primer año del gobierno de Andrés Manuel López Obrador (2018-2024), la evaluación de la educación en México experimentó un cambio muy significativo: la extinción del Instituto Nacional para la Evaluación de la Edu-

${ }^{7}$ Matías Busso et al. (eds.), Aprender mejor. Políticas públicas para el desarrollo de habilidades, Banco Interamericano de Desarrollo, 2017, https:// www.iadb.org/es/investigacion-y-datos/dia-2017-aprender-mejor-politi cas-publicas-desarrollo-habilidades (consulta del 21 de junio de 2019).

${ }^{8}$ Felipe Martínez Rizo y Emilio Blanco, "La evaluación educativa en México: experiencias, avances y desafíos", en Alberto Arnaut y Silvia Giorguli (coords.), Los grandes problemas de México VII. Educación, México, El Colegio de México, 2010, pp. 89-124. 
cación (INEE), el elemento clave de la evaluación de políticas educativas de los tres sexenios anteriores. ${ }^{9}$ Es crucial recapitular sobre la experiencia acumulada en el INEE durante sus 17 años de vida para recuperar lecciones sobre la evaluación de la educación que informen el proceso de diseño de las leyes y de los organismos a cargo de implementar la nueva reforma educativa.

Este artículo compara la manera en que se evaluaba la política educativa antes y después de que el INEE se convirtiera en un organismo constitucional autónomo (en 2013) y descubre la influencia persistente de la Secretaría de Educación Pública (SEP), así como la creciente participación de los gobiernos estatales. Para ello, se organiza en cinco secciones. La primera presenta el contexto político donde se inscribe este análisis y ocurren los cambios en la política de evaluación de la educación en México. Los siguientes cuatro apartados analizan en forma sistemática los cambios clave de la política de evaluación educativa en los últimos dos sexenios: la toma de decisiones, los actores, los objetos específicos de evaluación y los métodos en los temas de logro en aprendizajes, evaluación a docentes y programas federales de educación.

${ }^{9}$ El 15 de mayo de 2019 el gobierno de Andrés Manuel López Obrador promulgó la reforma educativa que derogó la de Enrique Peña Nieto y el 25 de septiembre del mismo año se aprobaron sus leyes secundarias. Los principales cambios fueron la sustitución del Sistema Nacional de Evaluación de la Educación (SNEE) por el Sistema Nacional de Mejora Continua de la Educación, la desaparición del Instituto Nacional para la Evaluación de la Educación (INEE) y la creación de la Comisión Nacional para la Mejora Continua de la Educación, la sustitución del Servicio Profesional Docente (SPD) por el Sistema de Carrera de las Maestras y Maestros, y la creación del Sistema Integral de Formación, Actualización y Capacitación. Véase "Los puntos clave de las tres leyes secundarias de la reforma educativa de AMLo", Animal Político, 20 de septiembre de 2019, https://www.animalpolitico.com/2019/09/claves-leyes-secundarias-reforma-educativa-amlo/ (consulta del 20 de noviembre de 2019). 
El CONTEXTO POLÍtico. LA REFORMA EDUCATIVA DE 2013

La reforma educativa del gobierno de Enrique Peña Nieto se aprobó en el Congreso, sin mayor discusión ni oposición partidista, bajo el manto del Pacto por México, como parte del grupo de once reformas estructurales que se aprobaron los primeros veinte meses de gobierno. ${ }^{10}$ La reforma modificó los artículos 3 y 73 de la Constitución mexicana con la finalidad de dotar al Sistema Educativo Nacional de los elementos necesarios para mejorar la calidad educativa y fortalecer la equidad. ${ }^{11}$

En lo que atañe al ciclo de políticas públicas, la reforma de 2013 identificó y definió causas precisas del problema de la baja calidad educativa para después diseñar acciones que atendían los problemas particulares de forma sistemática. Sin embargo, aunque se apegó a la teoría de las políticas públicas en cuanto a diseño, una serie de retos políticos y prácticos tergiversó su implementación y resultados. ${ }^{12}$

Durante todo el proceso de diseño e implementación predominó la percepción de que la reforma no era educativa, sino laboral. Esta percepción se construyó a partir de la combinación de cuatro factores: (i) la prensa, que desempeñó un papel muy importante como un actor político más en la ecuación, dando visibilidad a un puñado de temas sobre muchos

${ }^{10}$ Pedro Zepeda, El pacto por México; un intento de gobierno de coalición, Cuaderno de investigación 32, México, Instituto Belisario Dominguez, Senado de la República, 2017, http://bibliodigitalibd.senado.gob.mx/ bitstream/handle/123456789/3495/Cuaderno\%20de\%20investigación \%2032-1.pdf?sequence=1\&isAllowed=y (consulta del 24 de junio de 2019).

${ }^{11}$ Secretaría de Educación Pública (SEP), El libro blanco de la reforma educativa, México, SEP, 2018, https://www.gob.mx/sep/documentos/li bro-blanco-reforma-educativa-2013-2018-182474 (consulta del 20 de junio de 2019).

12 Óscar Faustino, "La reforma educativa de 2013 y lo que está por venir", Nexos, 26 de septiembre de 2018, https:/ / educacion.nexos.com.mx/ ?p=1520 (consulta del 29 e junio de 2019). 
otros también importantes; ${ }^{13}$ (ii) la prioridad sobresaliente que la autoridad otorgó a la evaluación del desempeño docente; (iii) la pérdida de poder que sufrió el SNTE y la CNTE sobre el sistema educativo, producto de la reconfiguración de los actores y del encarcelamiento de su líder Elba Esther Gordillo; y (iv) la incertidumbre de la base trabajadora en cuanto al servicio profesional docente, pues se reclamaban la pérdida de estabilidad en sus trabajos y las afectaciones a sus derechos laborales. ${ }^{14}$

Para que cualquier reforma de gran impacto social pueda concretarse, es indispensable que responda no sólo a los diagnósticos de quienes la decretan, sino también a las necesidades y aspiraciones profesionales de los actores encargados de implementarla. ${ }^{15}$ La reforma educativa de 2013, sin embargo, se emprendió sin el profesorado y simplificando la relación entre el control de ciertas prácticas negativas, como la herencia de plazas y la calidad educativa. ${ }^{16}$ La reforma educativa partió del supuesto de que, de manera casi autónoma, un magisterio mejor preparado (en la práctica esto sólo significaba mejores resultados en evaluaciones estandarizadas) aumentaría la calidad de todo el sistema. De esta manera, la responsabilidad se trasladó a un solo actor, lo cual se sumó a la construcción histórica de una imagen de los maestros como personas mal preparadas, incapaces e ignorantes. ${ }^{17}$

${ }^{13}$ Gloria del Castillo, "El papel de la prensa en la reforma educativa: ¿informa, distrae o distorsiona?”, Nexos, 27 de septiembre de 2017, https:// educacion.nexos.com.mx/?p=670 (consulta del 29 e junio de 2019).

${ }^{14}$ Óscar Faustino, art. cit.

${ }^{15}$ José María González, "CNTE: la demanda de diálogo y la sordera de la SEP", Nexos, 24 de junio de 2016, https:/ / educacion.nexos.com.mx/?p= 266 (consulta del 29 de junio de 2019).

${ }^{16}$ Emilio Blanco, "Cómo educar sin maestros", Nexos, 16 de septiembre de 2015, https:/ / educacion.nexos.com.mx/?p=24 (consulta del 29 de junio de 2019).

${ }^{17}$ Jorge Cano, "Sobre el fracaso de la reforma educativa: entrevista a Manuel Gil Antón”, Horizontal, 6 de junio de 2016, https:/ /horizontal.mx/ sobre-el-fracaso-de-la-reforma-educativa-entrevista-a-manuel-gil-anton/ (consulta del 29 de junio de 2019). 
Lo anterior agudizó un problema histórico del sistema educativo: la desconfianza entre docentes y autoridades hasta que el conflicto escaló, predominando el desprecio, la descalificación, la minimización y la violencia policial, en particular en contra de la CNTE. ${ }^{18}$

La implementación empezó por los aspectos laborales de la reforma -la evaluación del desempeño docente- y reservó para 2016 la parte educativa -el nuevo modelo educativo que se implementó a partir de 2018-. En ese momento, a pesar de todo lo que se había gastado en publicidad, las imágenes del gobierno federal y de la reforma se habían desgastado de manera considerable. ${ }^{19}$ De hecho, Carlos Ornelas señala las fallas de comunicación sobre el alcance de la reforma como la principal causa de incertidumbre, desconfianza y temor. ${ }^{20}$ Además, a pesar de la notoria experiencia de las autoridades del INEE, de su prestigio académico y su elevado desempeño técnico, el instituto no tuvo tiempo de sumar la legitimidad necesaria para convertirse en un interlocutor válido entre las partes en conflicto. ${ }^{21}$

El gran problema de la reforma educativa no fue su diseño o la parte técnica de la política pública, sino el aspecto político de su implementación. En breve, predominaron los logros políticos sobre los educativos. ${ }^{22}$ Aunque la reforma incluía muy diversos temas organizados en torno a siete prioridades, la atención pública que recibió la reforma giró sobre todo en torno al IneE y a su relación con el Servicio Profesional Docente.

${ }^{18}$ José María González, art. cit.

19 Óscar Faustino, art. cit.

${ }^{20}$ Carlos Ornelas, "De Oaxaca para Nuevo León”, Educación Futura, 6 de julio de 2016, http://www.educacionfutura.org/de-oaxaca-para-nue vo-leon/ (consulta del 9 de noviembre de 2019).

${ }^{21}$ Emilio Blanco, art. cit., y Luis Tapia, "Coaliciones y conflicto en torno a la reforma educativa: preservacionistas vs. evaluacionistas", Nexos, 24 de junio de 2016, https:/ / educacion.nexos.com.mx/?p=270 (consulta del 29 e junio de 2019).

22 Óscar Faustino, art. cit. 


\section{LAS DECISIONES GRUCIALES SOBRE LA EVALUACIÓN}

En este apartado se describe la manera en que el INEE se convirtió en la autoridad máxima del Sistema Nacional de Evaluación de la Educación y cómo se transformaron sus relaciones con la SEP, el Coneval y las entidades federativas.

En 2000, con la victoria de Vicente Fox, del Partido Acción Nacional y la alternancia política en el ejecutivo federal, especialistas de diversos campos prepararon los elementos para formar un nuevo programa de gobierno. El grupo encargado del área de evaluación incluyó la propuesta de crear un organismo técnico, con un grado considerable de autonomía, que se haría cargo de las evaluaciones del sistema educativo a gran escala. Hasta entonces, éstas habían sido responsabilidad exclusiva de la Dirección General de Evaluación de Políticas (DGEP) de la SEP. En la propuesta se denominó a esa instancia Instituto Nacional para la Evaluación de la Educación (INEE). ${ }^{23}$

La creación del INEe, con el decreto presidencial del 8 de agosto de 2002, destaca por dos avances en cuanto a la evaluación en el sector educativo. Primero, la evaluación de la educación cobró un papel imprescindible en la planeación y la rendición de cuentas de la autoridad educativa. Segundo, las instancias responsables de la evaluación se reestructuraron, pues antes de la creación del INEE, la Secretaría de Educación Pública (SEP) era el actor dominante en la evaluación, con cierta participación de las autoridades educativas estatales. ${ }^{24}$

${ }^{23}$ Felipe Martínez y Emilio Blanco, "La evaluación educativa en México: experiencias, avances y desafíos", en Alberto Arnaut y Silvia Giorguli (coords.), Los grandes problemas de México VII. Educación, México, El Colegio de México, 2010, pp. 89-124.

${ }^{24}$ Loc. cit., p. 10. y Paulo Santiago et al. , Reviews of Evaluation and Assessment in Education: Mexico 2012, París, ocDe, 2012, https://www.oecd. $\mathrm{org} / \mathrm{mexico} /$ Mexico\%20Review\% 20 of\% 20Evaluation \% 20and\%20As sessment\%20in\%20Education.pdf (consulta del 2 de noviembre de 2018). 
La reforma constitucional en materia educativa de 2013 estableció que la evaluación debía garantizar el derecho a una educación de calidad con equidad, además de garantizar su mejora continua. Con este objetivo, se creó el Sistema Nacional de Evaluación Educativa, regido por una Política Nacional de Evaluación de la Educación y bajo el liderazgo del INEE. 25

El Sistema Nacional de Evaluación Educativa (SNEE) fue un conjunto orgánico y articulado de instituciones, procesos, instrumentos y acciones que distribuyó y coordinó las tareas entre los diversos actores que participan en el diseño y la implementación de la evaluación educativa, desde las autoridades federales en la SEP y el INEE, hasta las autoridades estatales. ${ }^{26}$

El siguiente cuadro sintetiza las principales actividades de los actores que participaron en la implementación de la evaluación de la política educativa, así como sus principales actividades en el periodo 2013-2018.

A partir de la reforma de 2013, el Instituto Nacional para la Evaluación de la Educación se convirtió en la autoridad en materia de evaluación de la política educativa y se encargó de diseñar y realizar las mediciones de los componentes, procesos y resultados del Sistema Educativo Nacional (SEN), expedir los lineamientos a los que se sujetarían las autoridades educativas para llevar a cabo las funciones de evaluación que les correspondían, generar y difundir información y,

${ }^{25}$ Instituto Nacional para la Evaluación de la Educación (INEE), Política Nacional de Evaluación de la Educación, 2015, en: http:/ / publicacio nes.inee.edu.mx/buscadorPub/P1/E/302/P1E302.pdf (consulta del 20 de junio de 2019).

${ }^{26}$ Loc. cit.; Teresa Bracho, "Una mirada de la reforma educativa", en Pedro Salazar, Arturo Oropeza y José Romero (coords.), México 2018. La responsabilidad del porvenir, tomo I, México, IDIC/unam/El Colegio de México, 2018, pp. 485-508 y Decreto por el que se expide la Ley del Instituto Nacional para la Evaluación de la Educación, artículos 11 y 12, Diario Oficial de la Federación, 11 de septiembre de 2013, http:/ / www.dof.gob.mx/ nota_detalle.php?codigo $=5313842 \&$ fecha $=11 / 09 / 2013$ (consulta del 27 de septiembre de 2017). 


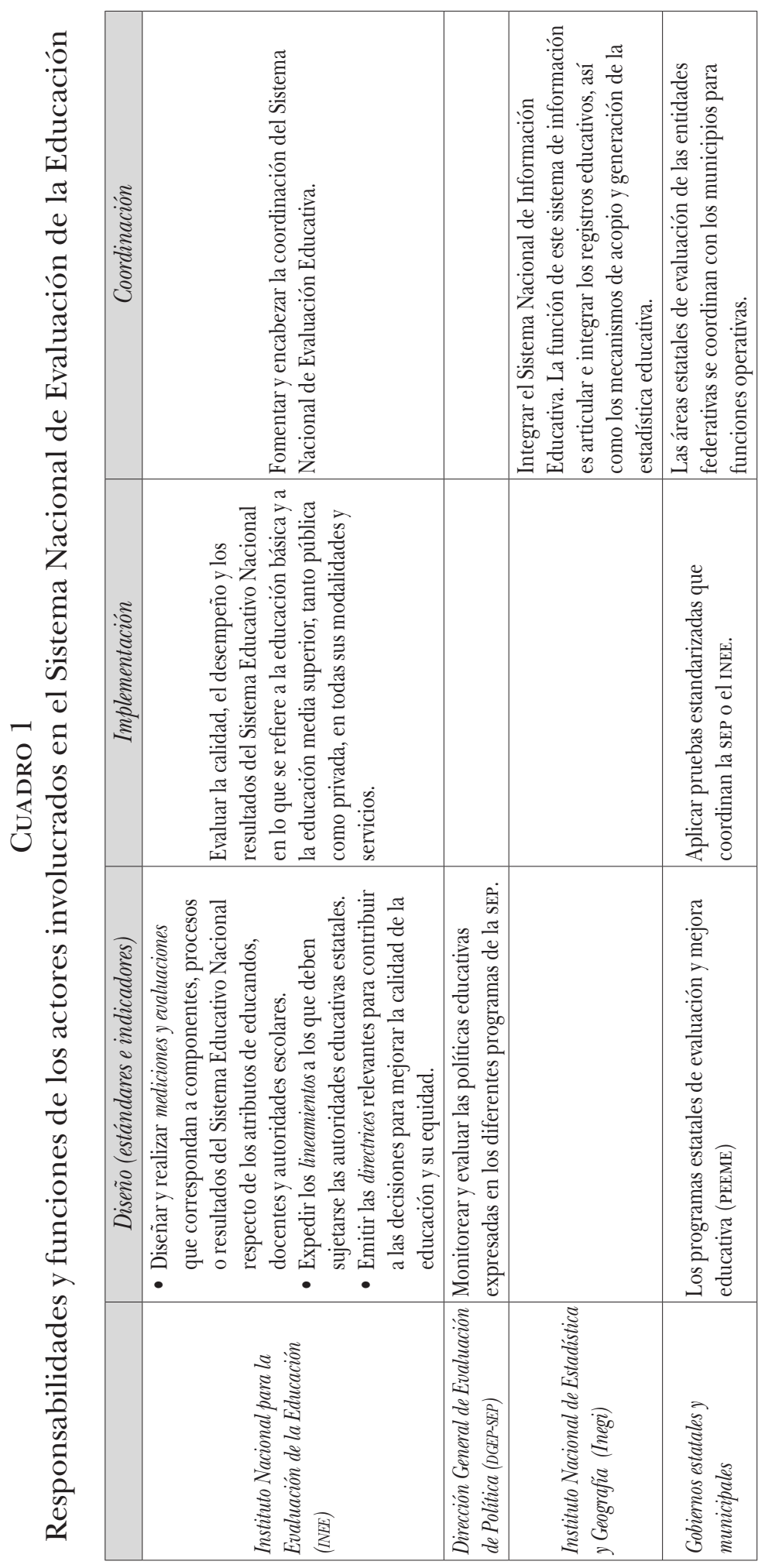




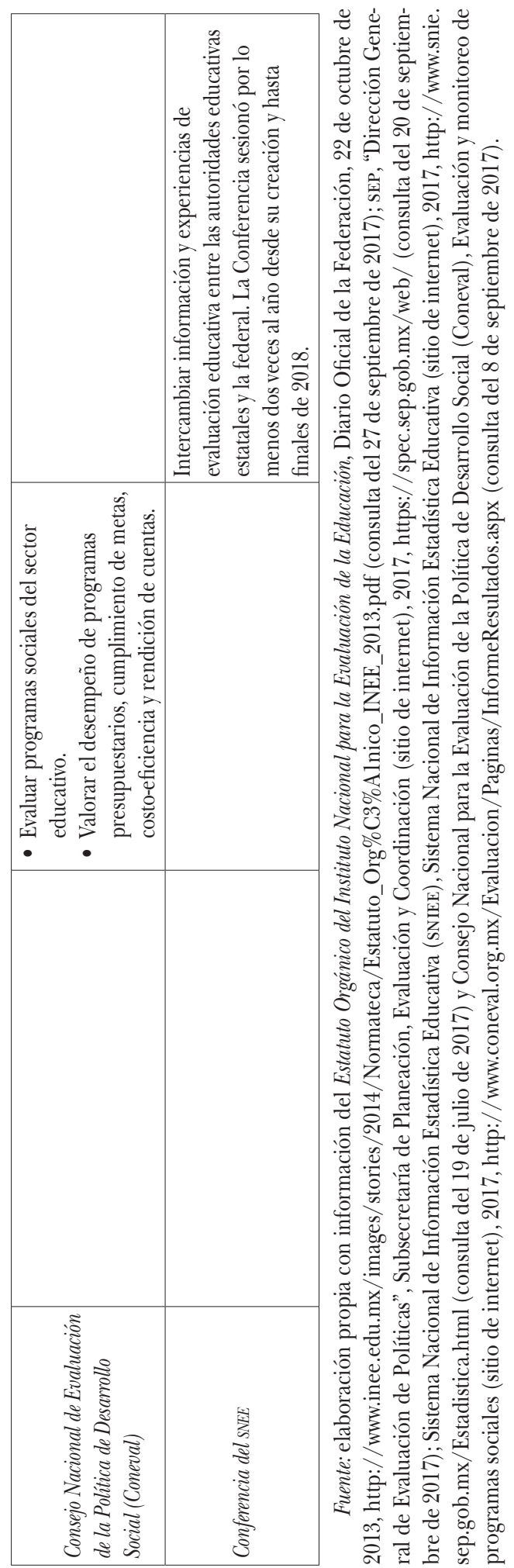


con base en ésta, emitir directrices para contribuir a las decisiones tendientes a mejorar la calidad de la educación y su equidad. 27

No obstante, la implementación de la evaluación era una actividad concurrente del INEE y de las autoridades educativas, tanto federales como estatales. El INEE estuvo obligado a establecer reglas de interacción entre los diversos actores que participan en el SNEE, pues se encontraba adscrito a un modelo de autoridad de traslape. ${ }^{28}$ Además, adquirió una importante función reguladora, pues las otras autoridades con atribuciones de evaluación estaban obligadas a atender los lineamientos emitidos por el Instituto. ${ }^{29}$

El documento rector de la Política Nacional de la Evaluación de la Educación se asumía como resultado del diálogo entre los distintos actores e instancias que participan en el SNEE bajo la convocatoria del INEE. Se esperaba que este documento rector presentara una visión de largo alcance para garantizar el derecho a la educación de calidad; además, significó un nuevo pacto entre el INEE y las autoridades educativas tanto del ámbito federal como del local. ${ }^{30}$

A partir de 2013, en esa nueva etapa de la evaluación de la educación, el INEe no tuvo una relación de subordinación con el gobierno federal o sus instancias, aunque su autonomía estuvo restringida por las normas que aplican a otros organismos en cuanto a presupuesto, responsabilidad de funcionarios públicos y transparencia. Antes de la reforma, el Instituto parecía estar encerrado en sí mismo, sin embargo, tras la auto-

27 Arcelia Martínez, "El InEE: Un nuevo actor en la política educativa", en Arcelia Martínez y Alejandro Navarro (coords.), La reforma educativa a revisión: apuntes y reflexiones para la elaboración de una agenda educativa 20182024, México, 2018. Instituto Belisario Domínguez/Senado de la República, pp. 197-220, 2018.

28 Teresa Bracho y Margarita Zorrilla, "Alcances, avances y retos de la autonomía del Instituto Nacional para la Evaluación de la Educación”, $R e-$ vista de Administración Pública del INAP, núm. 138 (septiembre-diciembre), 2015.

29 Teresa Bracho, art. cit.

${ }^{30}$ INEE, op. cit., 2015. 
nomía, comenzó a dialogar como par de las autoridades educativas federal y estatales, y a compartir información de manera más sistemática. ${ }^{31}$ En el periodo 2013-2018, el INEE mantuvo, por razones de diseño institucional aunque también prácticas, una relación de coordinación y colaboración con la SEP, así como con el Congreso federal y las autoridades educativas estatales.

Como ya mencionamos, al INEE le correspondió expedir los lineamientos que establecen las funciones de evaluación de cada una de las autoridades educativas. Esto incluyó uno de los elementos más controvertidos de la reforma de 2013 y del Instituto: definir los criterios técnicos para validar los parámetros e indicadores del perfil docente y directivo, así como establecer los lineamientos de la Coordinación Nacional del recién creado Servicio Profesional Docente (SPD). ${ }^{32}$

La tarea del SPD era ordenar la profesionalización del magisterio y construir un nuevo sistema basado en el mérito que garantizara la calidad de la educación, mejorara la práctica profesional docente y asegurara, con base en la formación continua y la evaluación, la idoneidad de los conocimientos y capacidades del magisterio. Al INEE le correspondió definir, regular y vigilar los procesos de evaluación del SPD; a la SEP determinar los criterios específicos y proveer los elementos necesarios para el desarrollo de los procesos de evaluación. Finalmente, a las autoridades educativas locales y a los organismos descentralizados operar, en sus respectivos ámbitos de competencias, las evaluaciones del personal docente, técnico docente, y del que desempeña actividades de dirección, supervisión y asesoría técnico-pedagógica. ${ }^{33}$

31 Teresa Bracho y Margarita Zorrilla, art. cit.

32 Arcelia Martínez, art. cit. y Jéssica Flores, "La formación de los docentes de educación media superior en México: el debate pendiente", en Arcelia Martínez y Alejandro Navarro (coords.), La reforma educativa a revisión: apuntes y reflexiones para la elaboración de una agenda educativa 20182024, México, Instituto Belisario Domínguez/Senado de la República, pp. 127-144.

33 SEP, op. cit., 2018 b. 
En suma, las instancias encargadas de la evaluación se reestructuraron. Antes de 2013, la seP y el Consejo Nacional para la Evaluación de la Política de Desarrollo Social (Coneval) eran los actores dominantes en la evaluación del personal docente y de programas, con la participación de las autoridades educativas estatales. El INEE era, sobre todo, un organismo técnico muy especializado que se ocupaba de las evaluaciones de logro educativo. Después de la reforma de 2013, en cuanto a las decisiones cruciales sobre evaluación, el INEE se convirtió en la autoridad principal en la política educativa.

\section{¿Quién EVALÚa?}

En este apartado, describimos los cambios en el INEE a partir de la reforma de 2013, con atención a su liderazgo, estructura y presupuesto. También analizamos las transformaciones que experimentó el papel de las entidades federativas en la evaluación de la política educativa.

Desde su creación, el INEE sufrió modificaciones diversas que le otorgaron las facultades necesarias para cumplir con su mandato de manera efectiva. En una primera etapa, de 2002 a 2012, operó como un organismo descentralizado de la SEP y, en una segunda, de 2012 a 2013, como descentralizado no sectorizado. A partir del 26 de febrero de 2013, el INEE se convirtió en un organismo público autónomo con personalidad jurídica y patrimonio propio ${ }^{34}$ para diseñar e implementar una política de evaluación educativa más allá de los límites de las administraciones sexenales. ${ }^{35}$

La modificación constitucional de 2013 reconoció que la evaluación educativa es un instrumento central para generar conocimientos que sirvieran como guía para la política

34 InEe, Tres años, 32 Programas Estatales de Evaluación y Mejora Educativa: balance 2016, en prensa(a).

35 Teresa Bracho, art. cit. 
sectorial y la gama de actores que participan en ella. ${ }^{36} \mathrm{El}$ INEE se convirtió en el líder del SNEE y su tarea principal era evaluar la calidad, el desempeño y los resultados del Sistema Educativo Nacional en la educación preescolar, primaria, secundaria y media superior. Sus funciones centrales eran: (a) técnica, al diseñar y realizar las mediciones que correspondan a componentes, procesos o resultados del sistema; (b) normativa vinculante, al expedir los lineamientos que sujetan a las autoridades educativas federal y locales para realizar las evaluaciones que les correspondan; (c) de comunicación, al generar y difundir información, y (d) de políticas, al emitir directrices con base en evidencia que permitan mejorar la calidad de la educación y su equidad. ${ }^{37}$

En cuanto a logro escolar del estudiantado, después de la reforma de 2013, el INEE tuvo un papel dominante en las evaluaciones estandarizadas con la colaboración de la SEP y las autoridades educativas estatales, mientras la sociedad y la prensa participaron de manera creciente en la difusión y discusión pública de los resultados de estas evaluaciones. ${ }^{38} \mathrm{Sin}$ embargo, el cambio más importante tras la autonomía del INEE en 2013 fue que transitó de ser un órgano concentrado en la evaluación del logro de aprendizajes a uno más visible, con más responsabilidades y con una expansión exacerbada en el aspecto técnico. ${ }^{39}$

A partir de la autonomía y con el mandato de emitir directrices generales para orientar la política educativa nacional, el Instituto comenzó a realizar evaluaciones integrales de los programas en operación que comparten un objetivo público específico: abatir la deserción escolar, mejorar la calidad de las escuelas multigrado o aumentar la inclusión de las personas indígenas en el sistema educativo. ${ }^{40}$ La evalua-

${ }^{36}$ Loc. cit. y Arcelia Martínez, art. cit., 2018.

37 Teresa Bracho, art. cit.

38 IneE, Política Nacional..., op. cit.

${ }^{39}$ Emilio Blanco, entrevista con las autoras, Ciudad de México, 1 de febrero de 2018.

${ }^{40}$ IneE, Política Nacional..., op. cit. 
ción de estos programas se definía en los planes anuales de evaluación que se formalizaban con la Secretaría de Hacienda, y que obligaba al Instituto a crear consensos para asignar responsabilidades entre la SEP y el Coneval.

Los cambios y el aumento en las funciones del Instituto significaron transformaciones en su liderazgo, estructura y presupuesto, y tuvieron como consecuencia cierta desorganización y burocratización en su funcionamiento en casos específicos. ${ }^{41}$

Los cambios en el liderazgo, la estructura y el presupuesto del INEE a partir de 2013

Como organismo descentralizado, antes de 2013, una junta directiva fungía como órgano de gobierno del InEE. Esta junta se designaba por decreto presidencial y se integraba con 14 personas: el presidente (secretario de Educación Pública) y 13 consejeros. El ejecutivo federal, la sEP o la junta directiva nombraban al director o directora general, quien debía tener experiencia en evaluación educativa y lideraba el Instituto por un periodo de cuatro años, con posibilidades de relegirse uno más. Además, la junta directiva el InEE designaba a un consejo técnico integrado por 16 personas expertas en el área de evaluación o investigación educativa, quienes duraban hasta ocho años en su cargo; cada año se sustituía a una cuarta parte de los integrantes. ${ }^{42}$

A partir de 2013, con la autonomía, los cinco integrantes de la junta de gobierno se elegían por el voto favorable de dos terceras partes de los senadores o la comisión permanente del Congreso de la Unión, a partir de ternas que proponía la Presidencia de la República. La junta de gobierno nombraba

${ }^{41}$ Emilio Blanco, art. cit., 2018.

${ }^{42}$ Estatuto orgánico del Instituto Nacional para la Evaluación de la Educación, Diario Oficial de la Federación, 31 de enero de 2017, http://www. diputados.gob.mx/LeyesBiblio/regla/n272.pdf (consulta del 27 de septiembre de 2017). 
a quien la presidiría con el voto de tres de sus integrantes, que eran personas con amplio reconocimiento en el sector por su experiencia en evaluación o investigación educativa. ${ }^{43}$

El INEE experimentó cambios muy sustanciales en sus funciones y liderazgo, como ya hemos detallado, pero también en su estructura y presupuesto. El número de plazas aumentó de 210, en 2012, a 550 en 2013; además, si consideramos las contrataciones eventuales, temporales y por honorarios, se registraron 2010 plazas en 2017. Este aumento tan sustancial se asocia con el número de evaluaciones de logro, y estudios nacionales e internacionales que realizaba el Instituto. ${ }^{44}$

En cuanto al presupuesto, también con datos del INEE, se observa un aumento muy sobresaliente de casi 975 millones de pesos, en términos constantes, entre 2013 y 2016. Los rubros que más aumentaron fueron los servicios personales, que incluyen la nómina del Instituto, y los generales. En agregado, el presupuesto del INEE aumentó 12 veces en términos reales en este periodo. En 2013, el INEE realizó sus actividades mediante tres programas presupuestales; en 2016, ya operaba con siete. Uno de los programas constantes desde 2013 fue la Evaluación del Sistema Educativo Nacional, que ocupó un porcentaje considerable del presupuesto total en ese año, 83 por ciento. El presupuesto para la Evaluación del SEN aumentó de manera destacada, poco más de 327 millones de pesos entre 2013 y 2017. En 2017, sin embargo, la Evaluación del Sistema Educativo ya sólo erogó 32 por ciento por el considerable incremento en el presupuesto global del INEE y porque se sumaron dos programas de alto gasto: Normatividad y política pública (26 por ciento) y Actividades de apoyo administrativo (23 por ciento). ${ }^{45}$

${ }^{43}$ Loc. cit.

${ }^{44}$ Laura Flamand y Claudia Santizo, Las politicas educativas fuera del centro. La evaluación como instrumento para promover la articulación y la igualdad en países multinivel, México, El Colegio de México, 2019, en dictamen, con base en los informes del ejercicio del gasto 2013-2017.

${ }^{45}$ Loc. cit., con base en los informes de actividades, de ejercicio del gasto y de gestión del INEE 2013-2017. 


\section{La participación de los gobiernos estatales}

Desde 2006 y hasta la autonomía del INEe en 2013, los gobiernos de las entidades federativas realizaron diversas actividades en materia de evaluación. En algunos estados se crearon institutos que sustituyeron a las áreas estatales de evaluación creadas desde la década de 1990 (Chiapas); en otras entidades, los institutos y las áreas estatales de evaluación se complementaron (Sonora, Nuevo León, Estado de México). Todas las entidades participaron en las evaluaciones nacionales de logro, tanto las nacionales que organizó la SEP o el INEE, como las internacionales. ${ }^{46}$

En 2016, como resultado de las reformas de 2013, las autoridades educativas locales desarrollaron los programas estatales de evaluación y mejora educativa (PEEME), que eran instrumentos de política que orientaban sus proyectos específicos de evaluación (Proeme), así como las acciones y metas principales en el mediano plazo, alrededor de 2020.

Los PEEME fueron el resultado de distintos espacios de interlocución entre los equipos técnicos de los estados y del INEE, así como entre los titulares de las secretarías de educación u homólogos de las entidades federativas y los consejeros de la junta de gobierno del Instituto, quienes se reunieron en varias ocasiones para discutir en perspectiva local, regional y nacional las propuestas de trabajo y las versiones definitivas de los programas. ${ }^{47}$

Se esperaba que, con los insumos de los PeEme y sus respectivos Proeme, fuera posible contar con información relevante: diagnósticos actualizados sobre el estado de la educación obligatoria en la entidad con atención a las brechas educativas de cada una, reportes de las capacidades institucionales desarrolladas para implementar los PEEME. Además, era indispensable dar cuenta de las formas de eje-

${ }^{46}$ Felipe Martínez y Emilio Blanco, art. cit.

47 InEe, Compendio de los Proyectos de Evaluación y Mejora Educativa (Proeme) de las 32 entidades federativas, México, en prensa. 
cución y de los resultados que se obtuvieron en cada uno de los Proeme. ${ }^{48}$

La transformación más importante del INEE con la reforma de 2013 es que transitó de un órgano concentrado en la evaluación del logro de aprendizajes a uno más visible, con más responsabilidades y una expansión muy considerable en el aspecto técnico. Esta transformación tan significativa supuso cambios en el liderazgo, la estructura y el presupuesto del INEE, y tuvo como consecuencia cierta burocratización en su operación. Antes de la reforma, todas las entidades participaban en las evaluaciones nacionales de logro, tanto las nacionales que organizó la SEP o el INEE, como las internacionales. Después de 2013, el papel de los gobiernos fue más amplio, incluyendo la planeación de la evaluación de la política, así como la articulación de la evaluación con el resto de las responsabilidades en materia educativa en el plano subnacional.

\section{¿QuÉ SE EVALÚA?}

En cuanto a los objetos a evaluar a partir de la autonomía, destacan tres conjuntos: las evaluaciones de logro de aprendizaje, de docentes y de programas. En esta sección detallamos los cambios en cada uno desde la reforma de 2013 que convirtió al INEe en un órgano constitucional autónomo.

\section{Evaluación de logro de aprendizaje}

A partir de 2006, la evaluación educativa en México tuvo avances significativos: un gran paso fue la puesta en marcha de la

48 INEE, Pautas para el acompañamiento de los Programas Estatales de Evaluación y Mejora Educativa, 2017, en: http:/ /www.inee.edu.mx/images/sto ries/2017/Gaceta7/suplementos/PAUTAS_27_de_junio.pdf (consulta del 6 de enero de 2018). 
Evaluación Nacional del Logro Académico en Centros Escolares, Enlace. Estas pruebas de tipo censal se aplicaron cada año desde 2006 y hasta 2013, y se emplearon para valorar el logro de todo el alumnado de los grados de tercero a sexto de primaria, los tres grados de secundaria y el último grado de educación media superior. Desde 2005, además, se empleó Excale (Exámenes de la Calidad y el Logro Educativo), una prueba muestral para evaluar el sistema educativo en conjunto. ${ }^{49}$

En 2014, ejerciendo sus nuevas funciones como órgano autónomo, el INEE sustituyó las pruebas Enlace y Excale por el Plan Nacional para la Evaluación de los Aprendizajes (Planea), con cambios sustanciales en objetivos, periodicidad y alcance. Las pruebas Planea fueron evaluaciones de diagnóstico de los resultados de aprendizaje en diferentes momentos de los niveles de educación..$^{50}$ Sus resultados ya no se empleaban para clasificar escuelas y alumnos según su desempeño (ranking), ni para evaluar el desempeño de docentes, como ocurrió con la prueba Enlace que servía como insumo en el programa Carrera magisterial. De 2014 a 2017 se aplicaron 36 evaluaciones de logro: SEP coordinó 18, el INEE 14 y ambas instituciones supervisaron 4 de manera conjunta. ${ }^{51}$

Entre los nuevos dispositivos de evaluación del INEE también se encontró la Evaluación de las Condiciones para la

${ }^{49}$ Felipe Martínez y Emilio Blanco, art. cit.

${ }^{50}$ Planea consideró tres tipos de evaluaciones: (1) La Evaluación del Logro del Sistema Educativo Nacional (ELSEN) que el INEE aplica en todo el país de manera censal al concluir primaria, secundaria y media superior. (2) La Evaluación del Logro de los Centros Escolares (ELCE) que la SEP aplica en coordinación con las autoridades educativas estatales al terminar primaria, secundaria y media superior. (3) La Evaluación Diagnóstica Censal (EDC) que los docentes frente a grupo aplican y califican a la mitad de la educación primaria. INEe, Plan Nacional para la Evaluación de los Aprendizajes (Planea). Documento Rector, México, INEE, 2018, http://pla nea.sep.gob.mx/content/general/docs/2018/PlaneaDocumentoRec tor18.pdf (consulta del 8 de octubre de 2018).

${ }^{51}$ Calendarios de evaluaciones 2014 a 2017, https://www.inee.edu. $\mathrm{mx} / 513$-reforma-educativa/1956-calendarios-sep-inee (consulta del $10 \mathrm{de}$ octubre de 2018). 
Enseñanza y el Aprendizaje (ECEA), que se propone valorar si los niños, independientemente de los aprendizajes, asisten a un espacio digno, bien equipado y con una comunidad escolar que propicie ambientes de respeto y convivencia armónica. En cuanto a los recursos para el aprendizaje, la evaluación consideró infraestructura, mobiliario y equipo, material y personal, mientras que en los procesos valoró la gestión del aprendizaje, la organización de la escuela y la convivencia escolar. ${ }^{52}$

\section{Evaluación docente}

Carrera magisterial fue un programa nacional de estímulos al personal docente de la educación básica que empezó en 1993 y se ajustó en diversos años hasta 2013, cuando se sustituyó por otra política de ingreso, promoción y permanencia. Ofreció un escalafón horizontal, de seis niveles salariales, que incrementaba los ingresos en el mismo cargo del personal directivo, docentes y técnico pedagógico. ${ }^{53}$ En sus lineamientos de 2011, el ingreso y la promoción de docentes en servicio consideraba la evaluación de cinco factores, de los cuales destacan dos: el aprovechamiento escolar de los alumnos de acuerdo con los resultados de Enlace $(50 \%)$ y la formación continua para incentivar la profesionalización de los docentes $(20 \%)$. La Comisión Nacional de Carrera Magisterial, integrada con representantes de la SEP y del SNTE, se ocupó de coordinar el ingreso y la promoción del programa. Aunque fue uno de sus objetivos, no hay evidencias de que hubiera una relación entre el ascenso en la carrera magisterial y la mejora de la calidad educativa. ${ }^{54}$

52 Arcelia Martínez, art. cit., 2018.

53 Secretaría de Educación Pública, sEP, Lineamientos Generales de Carrera Magisterial, México, Coordinación Nacional de Carrera Magisterial, 1998.

${ }^{54}$ Lucrecia Santibáñez et al., Haciendo camino. Análisis del sistema de evaluación y del impacto del programa de estímulos docentes Carrera Magisterial 
En 2008, cuando la SEP y el SNTE firmaron la Alianza por la Calidad de la Educación, se anunció que el acceso de los aspirantes a las plazas de maestro de educación básica ya no se decidiría de manera discrecional por las autoridades educativas o el sindicato, sino a partir de evaluaciones objetivas. ${ }^{55}$ La reforma de 2013 partió del diagnóstico de que la calidad de la enseñanza impartida en el aula es la principal causa del problema educativo. Por ello, el servicio profesional docente de 2013 concibió como solución la evaluación de docentes, técnicos docentes, supervisores, asesores técnico-pedagógicos y directivos en tres procesos -ingreso, promoción y permanencia en el servicio activo- con concursos de oposición para garantizar la idoneidad de conocimientos y capacidades. ${ }^{56}$

La Ley General del Servicio Profesional Docente (LGSPD) estableció el mecanismo para normar la trayectoria profesional del personal, así como los ejes fundamentales para fortalecer el desempeño del personal y, en consecuencia, la calidad del servicio educativo mediante la evaluación, la formación continua y el desarrollo profesional. La LGSPD fue, además, una disposición para sustituir el programa Carrera magisterial con un nuevo programa de incentivos, a partir del 31 de mayo de 2015; se trataba del Programa de Promoción en la Función por Incentivos en Educación Básica que incluía aplicar un examen de ingreso y una evaluación cada cuatro años. ${ }^{57}$

Por disposición constitucional, el INEE definía y supervisaba la evaluación docente, pues su carácter de organismo

en México, California, RAND Education, 2007, https://www.rand.org/con tent/dam/rand/pubs/monographs/2007/RAND_MG471.1.sum.pdf (consulta del 20 de junio de 2019).

${ }^{55}$ Felipe Martínez y Emilio Blanco, art. cit.

56 Decreto por el que se expide la Ley General del Servicio Profesional Docente, Diario Oficial de la Federación, 11 de septiembre de 2013, https:/ / www.dof.gob.mx/nota_detalle.php?codigo=5313843\&fecha=11/09/2013 (consulta del 20 de junio de 2019).

${ }^{57}$ Loc. cit. 
público autónomo aseguraba objetividad, transparencia e imparcialidad en su desarrollo. En 2014, se aplicaron tres evaluaciones a docentes que la SEP supervisó por completo, mientras que en 2015 se efectuaron siete en total, de las cuales SEP supervisó seis y el INEE sólo una. ${ }^{58}$ Estos datos revelan con relativa claridad que, a pesar de la imagen pública generalizada, la SEP siguió siendo el actor dominante en la evaluación docente.

\section{Evaluación de programas educativos}

Según el Inventario Coneval de programas federales de 2018, la valoración de los programas en el sector educativo es, a partir de diferentes criterios, el componente más importante en la evaluación de la política social que desarrolla el Consejo. Primero, el derecho social principal en el mayor número de programas sociales es la educación (52), seguido por bienestar económico (39) y salud (22). Segundo, la dependencia que opera más programas es la Secretaría de Educación Pública (38), después la Secretaría de Desarrollo Social (18) y la Secretaría de Salud (17). Tercero, por presupuesto dedicado, el derecho a la salud acumula el monto más elevado, con 375000 millones de pesos, seguido por educación, con 294.2 miles de millones de pesos. ${ }^{59}$

Entre 2012 y 2018, de acuerdo con la Secretaría de Educación Pública, se contrataron y supervisaron 55 evaluaciones a programas educativos: 16 de diseño, 21 de consistencia y resultados, y 18 específicas de desempeño. En todos los casos se trata de evaluaciones externas con un costo total de

58 Calendarios de evaluaciones 2014 a 2017, https://www.inee.edu. $\mathrm{mx} / 513$-reforma-educativa/1956-calendarios-sep-inee (consulta del $10 \mathrm{de}$ octubre de 2018).

${ }^{59}$ Coneval, Inventario de programas y acciones federales de desarrollo social, 2018, https://www.coneval.org.mx/Evaluacion/IPFE/Paginas/Default. aspx (consulta del 1 de noviembre de 2018). 
17.2 millones de pesos que se cubrió con recursos fiscales de SEP y Coneval. ${ }^{60}$

\section{¿Cómo se evalúa?}

Desde la reforma de 2013 se observaron cambios paulatinos en el número y la naturaleza de las evaluaciones del Coneval, el INEE y la SEP. Desde 2013, el INEE diseñó, contrató y supervisó más evaluaciones, compartió un calendario de evaluación con la SEP y se coordinó con los institutos estatales para realizar evaluaciones de logro. ${ }^{61}$ La participación de todos los actores fue compleja, pues el régimen concurrente los obligaba a cooperar para implementar las distintas evaluaciones. La SEP siguió participando activamente en la evaluación, no sólo por el número de evaluaciones que contrató y supervisó, sino por los recursos financieros que destinó a esta actividad.

En la práctica ocurrió una separación de funciones de evaluación entre los actores involucrados. Por un lado, el INEE, como líder del SNEE, se encargó junto con la SEP de las evaluaciones de logro educativo, docentes, condiciones de las escuelas, así como pruebas internacionales. ${ }^{62}$ Entre 2014 y 2018 el INEE efectuó 26 de estas evaluaciones; la SEP, 23, y de manera conjunta hicieron dos. Los años con más evaluaciones fueron 2015 y 2018, con 13, y el que menos tuvo fue 2017, con seis evaluaciones, debido a que ese año no se evaluó a los docentes. Esto revela qué tan prominente siguió siendo el papel de la SEP en términos de evaluación del sEN. ${ }^{63}$

${ }^{60}$ Laura Flamand y Claudia Santizo, op. cit.

${ }^{61}$ Loc. cit.

${ }^{62}$ Sylvia Schmelkes, entrevista con las autoras, Ciudad de México, 7 de febrero de 2018.

${ }^{63}$ Calendarios de evaluaciones 2014 a 2017, https://www.inee.edu. $\mathrm{mx} / 513$-reforma-educativa/1956-calendarios-sep-inee (consulta del 10 de octubre de 2018). 
Por otro lado, el Coneval, junto con la SEP, se encargó de las evaluaciones de programas presupuestales de educación. Entre 2012 y 2018, la SEP financió 16 evaluaciones de diseño y 21 de consistencia y resultados, con una inversión total de alrededor de 15 millones de pesos. En el mismo periodo, el Coneval financió 18 evaluaciones específicas de desempeño, con una inversión de alrededor de 2 millones de pesos. ${ }^{64}$

En su Programa Estratégico de Desarrollo Institucional 2015-2020, el INEE enfatizó la necesidad de fortalecer la comunicación y colaboración con el Coneval, sin embargo, el análisis de convenios de colaboración revela que estos intercambios no se materializaron, con la excepción muy notable del indicador de rezago educativo para la medición multidimensional de la pobreza. ${ }^{65}$

A partir de la reforma de 2013, una de las atribuciones del INEE fue emitir directrices elaboradas con base en el análisis de los resultados de las evaluaciones preparadas en el contexto del SNEE, de la investigación educativa disponible y de la revisión de las acciones públicas en educación, e intervienen en su elaboración docentes, directivos escolares, servidores públicos, académicos y representantes de organizaciones de la sociedad civil. Las directrices se dirigieron a las autoridades educativas, federal y locales, responsables de ejercer el presupuesto, así como de diseñar y desarrollar intervenciones para atender las necesidades educativas de la población. ${ }^{66}$

${ }^{64}$ Secretaría de Educación Pública, SEP, Evaluación Externa de Programas Federales, México, 2018, (https://www.gob.mx/sep/acciones-y-pro gramas/evaluacion-externa-de-programas-federales (consulta del 27 de octubre de 2018).

65 INEE, Programa Estratégico de Desarrollo Institucional 2015-2020, México, 2016, http://www.inee.edu.mx/images/banners/Transparencia_fracci \%C3\%B3n_VI/2016/4to_Trimestre_2016/PROEDI_0310161_2.pdf (consulta del 2 de noviembre de 2018).

66 INEE, Modelo para la construcción y emisión de directrices para la mejora educativa, México, INEE, 2015, http://www.inee.edu.mx/images/stories/ 2015/directrices/ruta.pdf (consulta del 10 de octubre de 2018). 
Las directrices fueron una nueva modalidad de evaluación, pues empleaban las evaluaciones publicadas de programas y otras investigaciones con un elevado rigor metodológico para informar la toma de decisiones de política, para mejorar la calidad de la educación y aumentar su equidad. En suma, las directrices aspiraban a que la política educativa se basara en evidencia. El mérito de las directrices se sustentaba en dos componentes: la valoración de los alcances y déficits de la acción pública, así como la construcción participativa. ${ }^{67}$ Desde 2015 y hasta 2018, el INEE emitió cinco conjuntos de directrices: 1) Para mejorar la formación inicial de los docentes de educación básica (2015); 2) Para mejorar la atención educativa de niñas, niños y adolescentes de familias de jornaleros agrícolas migrantes (2016); Para mejorar la atención educativa de niñas, niños y adolescentes indígenas (2017); Para mejorar la permanencia escolar en la educación media superior (2017), y Para mejorar la formación inicial de los docentes de educación básica (2018). ${ }^{68}$

La ruta para emitir una directriz tenía seis fases: construcción, emisión, comunicación, respuesta, implementación, monitoreo y actualización. Las primeras directrices se emitieron (segunda fase del proceso) relativamente hace poco tiempo, en 2015, de modo que cuando desapareció el INEE no había suficiente evidencia para determinar primero, si las agencias gubernamentales las estaban tomando en cuenta para guiar la acción pública y, segundo, si el hecho de que se desarrollen a partir de evaluaciones e investigaciones rigurosas en el problema específico aumentaba su efectividad.

El diseño de las directrices establecía que los gobiernos estatales respondieran a las propuestas elaborando una ruta

67 Arcelia Martínez, "Las directrices del INEE: construyendo puentes entre la evaluación, su uso y la mejora educativa”, Gaceta del INEE, núm 004 (2016), pp. 56-61, http://www.inee.edu.mx/images/stories/2016 / gaceta/ n\%C3\%BAmero_4/G04_ESP.pdf (consulta del 12 de octubre de 2018).

68 INEE, Directrices para mejorar la formación inicial de los docentes de educación básica, México, 2018, https://www.inee.edu.mx/index.php/proyec tos/directrices (consulta del 10 de octubre de 2018). 
de acción para implementarlas. No obstante, su puesta en marcha dependió, entre otros factores locales, de las capacidades de las autoridades de los estados y, además, se entrecruzó con problemas severos como la desigualdad. Además de consultar a los actores involucrados, las directrices tenían que mantener en la mira el contexto dinámico en el que viven y se desarrollan los beneficiarios directos de estas políticas. ${ }^{69}$

Finalmente, las directrices fueron una ruta para la mejora, pues colocaron problemas en la agenda pública, sin embargo, no fue posible vislumbrar su impacto. El hecho de que las autoridades educativas aceptaran las directrices fue un logro significativo, pues no eran vinculantes. Sin embargo, esperar que las directrices, incluso aquéllas respaldadas con evidencia rigurosa, generaran cambios por sí solas es poco realista, pues no existe una relación lineal entre evaluación y mejora. Las directrices hubieran logrado influir en las intervenciones públicas acompañadas de cabildeo y de seguimiento a las acciones que las autoridades educativas federales y estatales hubieran comprometido. ${ }^{70}$

En suma, en cuanto a la evaluación del logro escolar, en sus nuevas funciones como órgano autónomo a partir de 2013, el InEe sustituyó las pruebas Enlace y Excale con el Plan Nacional para la Evaluación de los Aprendizajes (Planea), con cambios sustanciales en objetivos, periodicidad y alcance. En cuanto a la evaluación del personal docente, Carrera magisterial era un programa nacional de estímulos a este personal que experimentó diversos ajustes desde su creación en 1993 y hasta 2013, cuando se sustituyó por el Servicio Profesional Docente, donde la SEP seguía siendo el actor dominante con una participación marginal del INEE. En cuanto a la eva-

${ }^{69}$ Ivania de la Cruz, "Directrices para la mejora de permanencia en educación media superior: ideas para alinear estrategias nacionales a contextos locales", Nexos, 21 de febrero de 2018, https://educacion.ne xos.com.mx/?p=1009 (consulta del 16 de octubre de 2018).

70 Arcelia Martínez, "Las directrices del INEE: ¿Una oportunidad para la mejora educativa?", Nexos, 15 de febrero de 2017, https://educacion. nexos.com.mx/?p=459 (consulta del 16 de octubre de 2018). 
luación de programas educativos, el Coneval siguió siendo la figura líder con una participación persistente de la SEP y una creciente del INEE. En particular, en el Instituto destacó el desarrollo de directrices para aumentar la calidad y la equidad en la educación.

\section{Conclusiones}

Como resultado de la reforma de 2013, es posible observar cambios importantes. El primero se refiere a la forma en que se toman las decisiones, en el periodo de 2006 a 2018. Entre 2006 y 2012, el INEE funcionó como organismo técnico, con un grado considerable de autonomía, elaborando evaluaciones del sistema educativo a gran escala. Las decisiones sobre la evaluación se negociaban con la SEP, en particular, con la DGEP. A partir de 2013, el INEE se convirtió en la autoridad máxima en materia de evaluación de la política educativa, pues era el organismo encargado de realizar las evaluaciones para todo el Sistema Nacional de Evaluación, y también de establecer los lineamientos y criterios de evaluación. A partir de la autonomía, en suma, la tarea principal del inEe fue evaluar la calidad, el desempeño y los resultados del Sistema Educativo Nacional en la educación preescolar, primaria, secundaria y media superior. Sin embargo, en cuanto a la evaluación de docentes, el Instituto cayó presa de la legalidad y del contexto político de la reforma: era un órgano técnico especializado al cual se le asignaron tareas políticas delicadas, por tanto, cometió serios errores de comunicación.

El segundo cambio se refiere a los actores que participan de la evaluación. A partir de que los tres objetos más relevantes de la evaluación fueron 1) logro, 2) docentes y 3) programas, observamos el cambio en la participación de los actores involucrados en las evaluaciones. Desde 2006 y hasta 2012, las evaluaciones de logro académico, básicamente pruebas estandarizadas para el estudiantado, eran facultad del INEE. Este tipo de evaluación no cambió después de la reforma de 
2013, pues el INEE siguió siendo responsable de realizarlas. La evaluación de docentes era facultad de la SEP, sin embargo, a partir de la reforma de 2013, el INEE también participó en estas evaluaciones. Entre 2006 y 2012, las evaluaciones de los programas educativos eran responsabilidad de la SEP y del Coneval, después de la reforma de 2013, la SEP, el INEE y el Coneval evaluaban los programas educativos. El InEe se involucró, sobre todo, con la intención de nutrir las directrices para aumentar la calidad y la equidad de los servicios educativos. Además, desde 2013, se promovió un papel creciente de las autoridades de evaluación estatales. Con la creación de las Direcciones del Instituto Nacional para la Evaluación de la Educación (DINEE) en los estados, el INEE apostó, en el largo plazo, por el federalismo, es decir, puso énfasis en fortalecer y coordinar a los actores que participan en la evaluación en las entidades. ${ }^{71}$

El tercer cambio refiere a lo que se evalúa de la política sectorial. De esta forma podemos observar que un paso importante para la evaluación de la política educativa fue que entre 2006 y 2012, el INEE y la sEP elaboraron el Sistema Nacional de Evaluación de manera conjunta, incluyendo la difusión de un primer anuario de indicadores para contribuir a la evaluación y al mejoramiento de la calidad de los subsistemas de educación básica y media superior. Los componentes que se evaluaban eran logro, docentes y programas. A partir de 2013, el logro académico de los estudiantes se siguió evaluando a través de pruebas estandarizadas. La evaluación de maestros en el SPD, que sustituyó a Carrera magisterial, incluyó la evaluación de docentes, técnicos docentes, supervisores, asesores técnico-pedagógicos y directivos en cuanto al ingreso, la promoción y la permanencia en el servicio activo con concursos de oposición para garantizar la idoneidad de conocimientos y capacidades. Además, el nuevo SNEE incluyó también la evaluación de los programas que atienden el derecho a la educación.

${ }^{71}$ Sylvia Schmelkes, Loc. cit. 
Finalmente, uno de los elementos más importantes de la reforma de 2013 fue que el INEE utilizó los resultados de estas evaluaciones para elaborar directrices que informaran las políticas educativas para mejorar la calidad y la equidad de la educación. Sin embargo, las directrices no fueron vinculantes ni incluyeron algún mecanismo que implicara consecuencias, pues se trataba de evaluaciones formativas, capacitantes.

\section{REFERENCIAS BIBLIOGRÁFICAS}

Blanco, Emilio, "Cómo educar sin maestros", Nexos, 16 de septiembre de 2015, https:/ / educacion.nexos.com.mx/?p=24 (consulta del 29 e junio de 2019).

Blanco, Emilio, entrevista con las autoras, Ciudad de México, 1 de febrero de 2018.

Bourdieu, Pierre y Jean-Claude Passeron, Reproduction in Education, Society and Culture, Londres, Sage Books, 1990.

Bracho, Teresa y Margarita ZorRILla, "Alcances, avances y retos de la autonomía del Instituto Nacional para la Evaluación de la Educación", Revista de Administración Pública del INAP (núm. 138), septiembre-diciembre.

Bracho, Teresa, "Una mirada de la reforma educativa”, en Pedro Salazar, Arturo Oropeza y José Romero (coords.), México 2018. La responsabilidad del porvenir, tomo I, México, IDIC/UNAM/ El Colegio de México, 2018, pp. 485-508.

Busso, Matías, Julian Cristia, Diana Hincapié, Julián Messina y Laura Ripiani, Aprender mejor. Políticas públicas para el desarrollo de habilidades, Washington, Banco Interamericano de Desarrollo, 2017, https://www.iadb.org/es/investigacion-y-datos/dia 2017-aprender-mejor-politicas-publicas-desarrollo-habilidades (consulta del 21 de junio de 2019).

Cano, Jorge, "Sobre el fracaso de la reforma educativa: entrevista a Manuel Gil Antón", Horizontal, 6 de junio de 2016, https:// horizontal.mx/sobre-el-fracaso-de-la-reforma-educativa-entre vista-a-manuel-gil-anton/ (consulta del 29 de junio de 2019). 
Castillo, Gloria del, ” El papel de la prensa en la Reforma Educativa: ¿informa, distrae o distorsiona?", Nexos, 27 de septiembre de 2017, en: https://educacion.nexos.com.mx/?p=670 (consulta del 29 e junio de 2019).

Consejo Nacional para la Evaluación de la Política de Desarrollo Social (Coneval), Evaluación y monitoreo de programas sociales, 2017, http://www.coneval.org.mx/Evaluacion/Paginas/Infor meResultados.aspx (consulta del 8 de septiembre de 2017).

Consejo Nacional para la Evaluación de la Política de Desarrollo Social (Coneval), Inventario de programas y acciones federales de desarrollo social, 2018, https://www.coneval.org.mx/Evaluacion/ IPFE/Paginas/Default.aspx (consulta del 1 de noviembre de 2018).

Cruz, Ivania de la, "Directrices para la mejora de permanencia en educación media superior: ideas para alinear estrategias nacionales a contextos locales", Nexos, 21 de febrero de 2018, https:/ / educacion.nexos.com.mx/?p=1009 (consulta del 16 de octubre de 2018).

Decreto por el que se expide la Ley del Instituto Nacional para la Evaluación de la Educación, artículos 11 y 12, Diario Oficial de la Federación, 11 de septiembre de 2013, http:/ /www.dof.gob. $\mathrm{mx} /$ nota_detalle.php?codigo $=5313842 \&$ fecha $=11 / 09 / 2013$ (consulta del 27 de septiembre de 2017).

Decreto por el que se expide la Ley General del Servicio Profesional Docente, Diario Oficial de la Federación, 11 de septiembre de 2013, https:/ /www.dof.gob.mx/nota_detalle.php?codigo=5 313843\&fecha=11/09/2013 (consulta del 20 de junio de 2019).

El Colegio de México, Desigualdades en México/2018, México, El Colegio de México, 2018.

Estatuto Orgánico del Instituto Nacional para la Evaluación de la Educación, Diario Oficial de la Federación, 22 de octubre de 2013, http://www.inee.edu.mx/images/stories/2014/Norma teca/Estatuto_Org\%C3\%A1nico_INEE_2013.pdf (consulta del 27 de septiembre de 2017).

Estatuto Orgánico del Instituto Nacional para la Evaluación de la Educación, 31 de enero de 2017, http://www.diputados.gob. 
mx/LeyesBiblio/regla/n272.pdf (consulta del 27 de septiembre de 2017).

Faustino, Óscar, "La reforma educativa de 2013 y lo que está por venir", Nexos, 26 de septiembre de 2018, https://educacion. nexos.com.mx/?p=1520 (consulta del 29 de junio de 2019).

Flamand, Laura y Claudia SANTizo, Las políticas educativas fuera del centro. La evaluación como instrumento para promover la articulación y la igualdad en países multinivel, México, El Colegio de México, 2019 (en dictamen).

Flores, Jessica, "La formación de los docentes de educación media superior en México: el debate pendiente”, en Arcelia Martínez y Alejandro Navarro (coords.), La reforma educativa a revisión: apuntes y reflexiones para la elaboración de una agenda educativa 2018-2024, México, Instituto Belisario Domínguez/Senado de la República, pp. 127-144.

GonzÁlez, José María, "CNTE: la demanda de diálogo y la sordera de la SEP”, Proceso. Distancia por tiempos, 24 de junio de 2016, en: https://educacion.nexos.com.mx/?p=266, consultado el 29 e junio de 2019.

Instituto Nacional para la Evaluación de la Educación, INEE, Compendio de los Proyectos de Evaluación y Mejora Educativa (Proeme) de las 32 entidades federativas, México (en prensa).

Instituto Nacional para la Evaluación de la Educación, INEe, Directrices para mejorar la formación inicial de los docentes de educación básica, México, 2018, https://www.inee.edu.mx/index.php/ proyectos/directrices (consulta del 10 de octubre de 2018).

Instituto Nacional para la Evaluación de la Educación, INEE, Modelo para la construcción y emisión de directrices para la mejora educativa, México, 2015, http:/ / www.inee.edu.mx/images/stories/2015/ directrices/ruta.pdf (consulta del 10 de octubre de 2018).

Instituto Nacional para la Evaluación de la Educación, INEe, Pautas para el acompañamiento de los Programas Estatales de Evaluación y Mejora Educativa, México 2017, http://www.inee.edu.mx/ images/stories/2017/Gaceta7/suplementos/PAUTAS_27_ de_junio.pdf (consulta del 6 de enero de 2018).

Instituto Nacional para la Evaluación de la Educación, INEe, Plan Nacional para la Evaluación de los Aprendizajes (Planea). Documen- 
to Rector, México, 2018, http://planea.sep.gob.mx/content/ general/docs/2018/PlaneaDocumentoRector18.pdf (consulta del 8 de octubre de 2018).

Instituto Nacional para la Evaluación de la Educación, IneE, Politica Nacional de Evaluación de la Educación, México, 2015, https:/ / www.inee.edu.mx/wp-content/uploads/2019/01/P1E302.pdf (consulta del 20 de junio de 2019).

Instituto Nacional para la Evaluación de la Educación, INEe, Programa Estratégico de Desarrollo Institucional 2015-2020, México, 2016, http://www.inee.edu.mx/images/banners/Transparen cia_fracci\%C3\%B3n_VI/2016/4to_Trimestre_2016/PROE DI_0310161_2.pdf (consulta del 2 de noviembre de 2018).

Instituto Nacional para la Evaluación de la Educación, INEe, Tres años, 32 Programas Estatales de Evaluación y Mejora Educativa: balance 2016, México (en prensa).

Lange, Fabian y Robert Topel, "The Social Value of Education and Human Capital", en Eric Hanushek y Finis Welch (eds.), Handbook of the Economics of Education, vol. 1, Países Bajos, Elsevier, 2006, pp. 459-509.

Ley del Instituto Nacional para la Evaluación de la educación, https://www.sep.gob.mx/work/models/sep1/Resource/ 558c2c24-0b12-4676-ad90-8ab78086b184/ley_instituto_nacio nal_evaluacion_educativa.pdf (consulta del 20 de junio de 2019).

"Los puntos clave de las tres leyes secundarias de la reforma educativa de AMLO", Animal Político, 20 de septiembre de 2019, https:/ / www.animalpolitico.com/2019/09/claves-leyes-secundarias-re forma-educativa-amlo/ (consulta del 20 de noviembre de 2019).

Martínez, Arcelia, "El InEE: Un nuevo actor en la política educativa”, en Arcelia Martínez y Alejandro Navarro (coords.), La reforma educativa a revisión: apuntes y reflexiones para la elaboración de una agenda educativa 2018-2024, México, Instituto Belisario Domínguez/Senado de la República, 2018, pp. 197-220.

Martínez, Arcelia, "Las directrices del IneE: ¿Una oportunidad para la mejora educativa?”, Nexos, 15 de febrero de 2017, https:/ / educacion.nexos.com.mx/?p=459 (consulta del 16 de octubre de 2018). 
Martínez, Arcelia, "Las directrices del ineE: construyendo puentes entre la evaluación, su uso y la mejora educativa”, Gaceta del INEE, núm 004 (2016), pp. 56-61, http:/ /www.inee.edu.mx/ima ges/stories/2016/gaceta/n\%C3\%BAmero_4/G04_ESP.pdf (consulta del 12 de octubre de 2018).

Martínez, Felipe y Emilio Blanco, "La evaluación educativa en México: experiencias, avances y desafíos", en Alberto Arnaut y Silvia Giorguli (coords.), Los grandes problemas de México VII. Educación, México, El Colegio de México, 2010, pp. 89-124.

Ornelas, Carlos, "De Oaxaca para Nuevo León", Educación Futura, 6 de julio de 2016, http://www.educacionfutura.org/de-oaxa ca-para-nuevo-leon/ (consulta del 9 de noviembre de 2019).

Ornelas, Carlos, "The Politics of Privatization, Decentralization and Education Reform”, en Joseph Zajda (ed.), Decentralization and Privatisation in Education. The Role of the State, Países Bajos, Springer, 2006, pp. 207-228.

Santiago, Paulo, Isobel McGregor, Deborah Nusche, Pedro Ravela y Diana Toledo, Reviews of Evaluation and Assessment in Education: Mexico 2012, París, ocde, 2012, https://www.oecd. org/mexico/Mexico\%20Review\% 20of\%20Evaluation \% 20 and\%20Assessment\%20in\%20Education.pdf (consulta del 2 de noviembre de 2018).

Santibáñez, Lucrecia, José Martínez, Ashlesha Datar, Patrick McEwan, Claude Messan y Ricardo Basurto, Haciendo camino. Análisis del sistema de evaluación y del impacto del programa de estímulos docentes Carrera Magisterial en México, California, RAND Education, 2007, https://www.rand.org/content/dam/rand/ pubs/monographs/2007/RAND_MG471.1.sum.pdf (consulta del 20 de junio de 2019).

Schmelkes, Sylvia, entrevista con las autoras, Ciudad de México, 7 de febrero de 2018.

Secretaría de Educación Pública, SEP, Lineamientos Generales de Carrera Magisterial, México, Coordinación Nacional de Carrera Magisterial, 1998.

Secretaría de Educación Pública, SEP, "Dirección General de Evaluación de Políticas", Subsecretaría de Planeación, Evaluación y 
Coordinación, 2017, https://spec.sep.gob.mx/web/ (consulta del 20 de septiembre de 2017).

Secretaría de Educación Pública, SEP, El libro blanco de la reforma educativa, México, sEP, 2018, https://www.gob.mx/sep/documen tos/libro-blanco-reforma-educativa-2013-2018-182474 (consulta del 20 de junio de 2019).

Secretaría de Educación Pública, seP, Evaluación Externa de Programas Federales, México, 2018, https://www.gob.mx/sep/accionesy-programas/evaluacion-externa-de-programas-federales (consulta del 27 de octubre de 2018).

Sistema Nacional de Información Estadística Educativa, SNIEE, Sistema Nacional de Información Estadística Educativa, México, 2017, http:/ /www.snie.sep.gob.mx/acerca_sniee.html (consulta del 19 de julio de 2017).

TAPIA, Luis, "Coaliciones y conflicto en torno a la reforma educativa: preservacionistas vs. evaluacionistas", Nexos, 24 de junio de 2016, https://educacion.nexos.com.mx/?p=270 (consulta del 29 e junio de 2019).

ZEPEDA, Pedro, El pacto por México; un intento de gobierno de coalición, cuaderno de investigación 32, Instituto Belisario Dominguez, Senado de la República, 2017, http:/ / bibliodigitalibd.senado. gob.mx/bitstream/handle/123456789/3495/Cuaderno $\% 20$ de\% 20investigación \% 2032-1.pdf? sequence $=1$ \&isAllowed $=y$ (consulta del 24 de junio de 2019). 
